\title{
CXCR4 protects bone marrow-derived endothelial progenitor cells against hypoxia through the PI3K/Akt signaling pathway
}

\author{
ZHENG-YU LIU ${ }^{1,2^{*}}$, QIU-XIA YANG ${ }^{3 *}$, YAN CAO $^{4,5}$, HONG-WEI PAN $^{1,2}$, JING-JING RONG $^{1,2}$, JING LING $^{3}$,

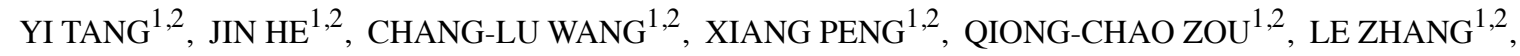 \\ JIAO ZHENG ${ }^{6}$, JIA WANG $^{6}$, YU ZHANG $^{1,2}$, JIAN-QIANG PENG $^{1,2}$, LAN-BING XIONG $^{3}$, \\ FENG LIU $^{3}$, ZI-HUI YING ${ }^{3}$, ZHAO-FEN ZHENG ${ }^{1,2}$ and BAI-LING ZHANG ${ }^{7}$
}

${ }^{1}$ Department of Cardiology, Hunan Provincial People's Hospital; ${ }^{2}$ Clinical Research Center for Heart Failure of Hunan Province, Department of Cardiology, Hunan Provincial People's Hospital; ${ }^{3}$ Department of Cardiology, The First Affiliated Hospital of Hunan Normal University (Hunan Provincial People's Hospital); ${ }^{4}$ Department of Emergency, Hunan Provincial People's Hospital; ${ }^{5}$ Emergency and Critical Care Metabolomic Key Lab of Hunan Province; ${ }^{6}$ Institute of Clinical Pharmacology Research, Hunan Provincial People's Hospital, Changsha, Hunan 410000;

${ }^{7}$ Department of Cardiology, Xiangxi Autonomous Prefecture People's Hospital, Jishou, Hunan 416000, P.R. China

Received January 3, 2021; Accepted May 21, 2021

DOI: $10.3892 / \mathrm{etm} .2021 .10634$

\begin{abstract}
The present study aimed to investigate the regulatory mechanism of chemokine (C-X-C motif) receptor 4 (CXCR4) on endothelial progenitor cells (EPCs) through the phosphatidylinositol 3-kinase/protein kinase B (PI3K/Akt) signaling pathway under hypoxic conditions. Mononuclear cells were isolated from the bone marrow (BM) of young Sprague-Dawley (SD) rats. Bone marrow-derived endothelial progenitor cells (BM-EPCs) were characterized by using Dil-labeled acetylated low-density lipoprotein (Dil-ac-LDL) and fluorescein isothiocyanate-labeled UEA (FITC-UEA-1). Phenotype identification of BM-EPCs was based on red cytoplasm and green cytomembrane. Flow cytometry was employed to examine the markers CD14, CD34, and KDR. Expression level of the EPC-specific surface marker CD14 was found to be negative, while the expression level of CD34 and KDR was positive. In addition, CXCR4 was stably overexpressed in BM-EPCs after transfection with
\end{abstract}

Correspondence to: Professor Zhao-Fen Zheng, Department of Cardiology, Hunan Provincial People's Hospital, 61 Jiefang Road, Changsha, Hunan 410000, P.R. China

E-mail: zhengzhaofen@hunnu.edu.cn

Dr Bai-Ling Zhang, Department of Cardiology, Xiangxi Autonomous Prefecture People's Hospital, 3 Jianxin Road, Jishou, Hunan 416000, P.R. China

E-mail: zhangbailing@163.com

${ }^{*}$ Contributed equally

Key words: CXC chemokine receptor type 4, bone marrow-derived endothelial progenitor cells, hypoxia, phosphatidylinositol 3-kinase, proliferation, migration, apoptosis
adenovirus-CXCR4. Cell proliferation, migration and apoptosis abilities were measured through the application of CCK-8, followed by Transwell and flow cytometry assays. The expression level of CXCR4, PI3K and Akt was determined by reverse transcription-quantitative PCR and western blotting assays. Functional experiments demonstrated that hypoxia inhibited BM-EPC proliferation and migration, while accelerating BM-EPC apoptosis. Additionally, CXCR4 was found to promote proliferation and migration, and suppress apoptosis in BM-EPCs with or without hypoxia treatment. Evidence also demonstrated that CXCR4 markedly upregulated the expression levels of PI3K and Akt. Furthermore, PI3K inhibitor (LY294002) and CXCR4 inhibitor (AMD3100) effectively inhibited the proliferation, migration and resistance to apoptosis of CXCR4-mediated BM-EPCs under hypoxic conditions.

\section{Introduction}

Cardiovascular disease (CVD) represents the most common cause of death worldwide (1). Endothelial dysfunction is an initial and aggravating factor of CVD; thus, an endothelial protection strategy could prevent and decrease the occurrence of CVD (2). Hypoxia is a common pathological condition that leads to vascular endothelial injury associated with a variety of CVDs, due to its ability to induce endothelial injury and dysfunction (3). Therefore, attenuation of hypoxia-induced vascular endothelial dysfunction is a promising strategy for the prevention and treatment of CVDs.

Endothelial progenitor cells (EPCs) are a group of stem cells that can specifically home to areas of angiogenic organization and differentiate into endothelial cells (4). EPCs as the central type of cell involved in the physiological and pathological processes of angiogenesis after birth (5). As precursors to vascular endothelial cells, EPCs are strongly implicated in influencing the stromal cells in the bone marrow (BM) (6) and 
in the occurrence of cardiovascular disease, cerebrovascular disease and tumor angiogenesis.

Chemokine receptor-4 (CXCR4) belongs to the G-protein coupled receptor (GPCR) family (7). The interactions between chemokines and chemokine receptors can induce the chemotactic migration of target cells, rearrangement of cell cytoskeleton, and intensive adhesive capacity between target cells and endothelial cells, which are extensively involved in biological functions such as cell growth, differentiation and apoptosis (8). Presently, it is known that one of the most important natural ligands of CXCR4 is stromal cell-derived factor-1 (SDF-1/CXCL12) (7). The combination of CXCR4 and SDF-1 can activate downstream signaling pathways including $\mathrm{Ca}^{2+}$ influx, phosphatidylinositol 3-kinase (PI3K), Akt, mitogen-activated protein (MAP) kinase, nuclear factor- $\kappa \mathrm{B}(\mathrm{NF}-\kappa \mathrm{B})$, proline-rich tyrosine kinase 2 and the CT10 regulator of kinase (Crk) pathway (9). The significant effects of SDF-1/CXCR4 interaction have been highlighted in the regulation of cellular functions including cell adhesion, cell proliferation, cell migration, cell survival and angiogenesis $(10,11)$. However, the functions and mechanisms of CXCR4 in BM-derived EPCs (BM-EPCs) have not previously been investigated.

The aim of the present study was to investigate the effects of CXCR4 overexpression on the proliferation, apoptosis and migration of hypoxia-treated BM-EPCs, and to explore the involvement of the downstream PI3K signaling pathway in these behaviors. BM-EPCs were pre-treated with hypoxia, in order to identify the regulatory mechanism of CXCR4 overexpression in EPCs under hypoxic conditions.

\section{Materials and methods}

Experimental animals. A total of 20 female Sprague Dawley (SD) rats (aged 4-6 weeks, weighing 200-240 g) were provided by Shrek (Shanghai, China). Animals were allowed free access to food and water, maintained in a 12-h light/dark cycle at room temperature $\left(21-25^{\circ} \mathrm{C}\right)$ in $50-60 \%$ relative humidity. Routine cleaning and disinfection were performed daily. The duration of the experiment was 10 months. Animal health was monitored every 3 months. The animals used in the present study were cared for in accordance with the Guide for the Care and Use of Laboratory Animals published by the National Institutes of Health, and methods were approved by the Animal Research Committee of Hunan Provincial People's Hospital [Changsha, China; approval no. (2019)-041].

Isolation and culture of EPCs. SD rats were anesthetized by intraperitoneal injection of $3 \%$ pentobarbital sodium $(30 \mathrm{mg} / \mathrm{kg}$ body weight) and then euthanized by means of cervical dislocation. Respiration and heartbeat were stopped to confirm the death of the animal, and the bodies were subsequently soaked in $75 \%$ ethanol solution for $15 \mathrm{~min}$. The femur and tibia were then separated under aseptic conditions, with the bone surface of the femur and tibia carefully removed and sheared accordingly. The BM cavity was repeatedly washed with low-glucose Dulbecco's modified Eagle's medium (L-DMEM; Beyotime Institute of Biotechnology), after which the flushing fluid was collected. The collected flushing fluid was incubated with the equivalent volume of lymphocyte separation medium (Wisent,
Inc.). The solution was centrifuged at $2,000 \mathrm{x} \mathrm{g}$ for $15 \mathrm{~min}$ at room temperature to collect the cells, which were seeded into a culture dish with $200 \mathrm{ng} / \mathrm{ml}$ fibrinolysin at a concentration of $1 \times 10^{6} / \mathrm{cm}^{2}$. The cells were then incubated in L-DMEM with $10 \%$ fetal bovine serum (FBS; Gibco; Thermo Fisher Scientific, Inc.) at $37^{\circ} \mathrm{C}$ with $5 \% \mathrm{CO}_{2}$.

Phenotype identification of BM-EPCs. The BM-EPCs were characterized by immunofluorescent staining and flow cytometry analysis. For immunofluorescent staining, the BM-EPCs were harvested and washed with phosphate buffered saline (PBS) and incubated with $200 \mu 1$ Acetylated DiI lipoprotein from human plasma (Dil-Ac-LDL; $10 \mu \mathrm{g} / \mathrm{ml}$; Molecular Probes; Thermo Fisher Scientific, Inc.) for $4 \mathrm{~h}$ at $37^{\circ} \mathrm{C}$ with $5 \% \mathrm{CO}_{2}$. Samples were then fixed with $4 \%$ paraformaldehyde for $30 \mathrm{~min}$ at $37^{\circ} \mathrm{C}$. Cells were stained with $200 \mu$ l fluorescein isothiocyanate labeled ulex europaeus lectin-1 (FITC-UEA-1; $10 \mu \mathrm{g} / \mathrm{ml}$; Sigma Aldrich; Merck KGaA) for $1 \mathrm{~h}$ at $37^{\circ} \mathrm{C}$ with $5 \% \mathrm{CO}_{2}$, followed by observation under a fluorescence microscope (magnification, x120; Olympus Corporation).

For flow cytometry analysis, the BM-EPCs were lysed in PBS (Invitrogen; Thermo Fisher Scientific, Inc.) with $0.5 \%$ paraformaldehyde (Sigma Aldrich; Merck KGaA) and 0.5\% saponin (Sigma Aldrich; Merck KGaA) for 5 min at $4^{\circ} \mathrm{C}$. The cells were labeled with rabbit anti-mouse FITC-CD14 (BD Biosciences), rabbit anti-mouse PE-CD34 (Bio Legend, Inc.), and rabbit anti-mouse PerCP/Cy5.5-KDR (BioLegend, Inc.) for $1 \mathrm{~h}$ at room temperature. The same fluorescein-labeled isotype $\mathrm{IgG}$ was used as a control to define the negative population for each stain. After washing, cells were analyzed with the FACS $^{\text {TM }}$ Canto II flow cytometer. CD14, CD34 and KDR were examined using the FACSDiva software (v.6; BD Biosciences) for fluorescence-activated cell sorting, based on the manufacturer's instructions.

Adenovirus transfection. The BM-EPCs at passage number 2 were used for transduction. Viral vector, packaging plasmid, adenovirus related vectors (rAAV6 vectorand pHelper1.0 vector) and 293 T cells were purchased from Cyagen Biosciences Inc. 293T cells were transfected by the calcium phosphate method. The recombinant plasmids pAAV2.1-CMV-GFP $(0.5 \mu \mathrm{g} / \mathrm{ml})$ or pAAV2.1-CMV-CXCR4 $(5 \mu \mathrm{g} / \mathrm{ml})$ and the packaging plasmid pRC6 $(2 \mu \mathrm{g} / \mathrm{ml})$ and pHelper $(10 \mu \mathrm{g} / \mathrm{ml})$ were added to $\mathrm{CaCl}_{2}$, then mixed with 2X HBS to make Calcium-DNA. 293T cells were then co-transfected with Calcium-DNA at $37^{\circ} \mathrm{C}$ with $5 \% \mathrm{CO}_{2}$. After $48 \mathrm{~h}$, the viral supernatant was harvested with centrifuged at $2,000 \mathrm{x} \mathrm{g}$ for $20 \mathrm{~min}$ at $4^{\circ} \mathrm{C}$. The concentration of recombinant adeno-associated virus was determined by reverse transcription-quantitative polymerase chain reaction (RT-qPCR). BM-EPCs (1x10\%/well) were seeded in 24-well cell culture plates and cultured overnight. Then, BM-EPCs were subsequently transfected with rAAV6-GFP and rAAV6-CXCR4 at the indicated multiplicity of infection (moi). After incubation at $37^{\circ} \mathrm{C}$ for $48 \mathrm{~h}$, the transfection efficiency of the lentivirus vectors in transduced-EPCs was identified using fluorescence microscope. The level of CXCR4 expression was detected by western blotting, RT-qPCR and flow cytometry.

Cell treatment. After the BM-EPCs were transfected with $\mathrm{rAAV}_{6}-\mathrm{GFP}$ and $\mathrm{rAAV}_{6}-\mathrm{CXCR} 4$, they were cultured at $37^{\circ} \mathrm{C}$ 
with $5 \% \mathrm{CO}_{2}$ in a humidified atmosphere with Endothelial Cell Growth Medium-2 (Lonza Group, Inc.), supplemented with $10 \% \mathrm{FBS}, 100 \mathrm{U} / \mathrm{ml}$ penicillin and streptomycin sulfate (Thermo Fisher Scientific, Inc.), and SDF-1 at $20 \mu \mathrm{g} / 1$ for conventional culture. Initially, the BM-EPCs in the normoxia group were cultured under normoxic conditions $\left(5 \% \mathrm{CO}_{2}, 95 \%\right.$ air atmosphere). BM-EPCs in the hypoxia group were cultured under anaerobic conditions $\left(5 \% \mathrm{CO}_{2}, 95 \% \mathrm{~N}_{2}\right)$ free of FBS for $4 \mathrm{~h}$, and then cultured under normoxic conditions with PBS for $6 \mathrm{~h}$. The BM-EPCs were subsequently divided into four groups: Normoxia + Sham (Empty vector) group, Normoxia + CXCR4 group, Hypoxia + Sham group, and Hypoxia + CXCR4 group. In additional experiments, the BM-EPCs were divided into these four groups: Hypoxia + Sham group, Hypoxia + CXCR4 group, Hypoxia + CXCR4 + PI3K inhibitor (LY294002, $20 \mu \mathrm{mol} / 1$ for $1 \mathrm{~h}$ ) group, and Hypoxia + CXCR4 + CXCR4 inhibitor (AMD3100) group.

Cell counting kit-8 (CCK-8) assay. The treated BM-EPCs $\left(2 \times 10^{3}\right.$ cells/well) were seeded into 96 -well plates at $37^{\circ} \mathrm{C}$. CCK-8 reagent (cat. no. CK04; Dojindo Molecular Technologies, Inc.) was added at the 24, 48, 72 and $96 \mathrm{~h}$ time points. After an additional $3 \mathrm{~h}$, the absorbance value was determined by a microplate reader (Multiscan MK3; Thermo Fisher Scientific, Inc.) at $450 \mathrm{~nm}$. The mean optical density (OD) of the three wells in each group was used to reflect the percentage of cell proliferation.

Transwell assay. The treated BM-EPCs $\left(5 \times 10^{4}\right.$ cells/well $)$ were added to the top of chambers (BD Biosciences) with serum-free medium. The complete medium was then added to the lower chambers. After $24 \mathrm{~h}$, the non-migrated cells were removed, while the migrated cells were fixed using $4 \%$ paraformaldehyde for $20 \mathrm{~min}$, and stained with $0.1 \%$ crystal violet solution for 5-10 min at $37^{\circ} \mathrm{C}$ (Sigma Aldrich; Merck $\mathrm{KGaA}$ ). Lastly, images were captured under a light microscope (magnification, $\mathrm{x} 200$ ). The experiment was performed three times.

Annexin V-FITC/PI double staining assay. The treated BM-EPCs $\left(1 \times 10^{6}\right.$ cells $\left./ \mathrm{ml}\right)$ were stained with Annexin V-FITC/PI (Multsciences) under dark conditions for $15 \mathrm{~min}$ at room temperature. Cell apoptosis was detected using a FACS Calibur flow cytometry (BD Biosciences) with CellQuest software (version FACS101; BD Biosciences). The experiment was performed three times.

RT-qPCR assay. The total RNA of the treated BM-EPCs was extracted using TRIzol reagent (cat. no. 9109; Takara Biotechnology Co. Ltd.). Then, $3 \mu \mathrm{g}$ RNA was reverse transcribed to cDNA using a cDNA synthesis kit (cat. no. 6130; Takara Biotechnology Ltd.) following the manufacturer's instructions. The expression levels of CXCR4 were detected using SYBR Green PCR Master Mix kit (Takara Biotechnology Co. Ltd.) on an ABI-Prism 7300 system (Applied Biosystems; Thermo Fisher Scientific, Inc.). $\beta$-actin was used as an endogenous control. Results were calculated using the $2^{-\Delta \Delta \mathrm{Cq}}$ method (12). The following primers were used: $\beta$-actin (product length, $150 \mathrm{bp}$ ) forward, 5'-GGAGATTAC TGCCCTGGCTCCTA-3'; $\beta$-actin reverse, 5'-GACTCATCG
TACTCCTGCTTGCTG-3'; CXCR4 (product length 113 bp) forward, 5'-CCATCCTCTACGCCTTCC-3'; CXCR4 reverse, 5'-TCCACCCCGTTTCCCTT-3'. The qPCR reaction mixture consisted of the following: $1 \mu \mathrm{l} \mathrm{cDNA}, 0.5 \mu \mathrm{l}$ forward primers and $0.5 \mu \mathrm{l}$ reverse primers, $10 \mu \mathrm{l}$ SYBR Green PCR Master

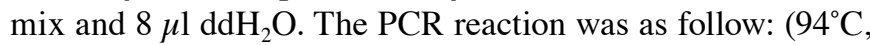
$2 \mathrm{~min} ; 94^{\circ} \mathrm{C}, 20 \mathrm{sec} ; 58^{\circ} \mathrm{C}, 20 \mathrm{sec} ; 72^{\circ} \mathrm{C}, 20 \mathrm{sec} ; 40$ cycles in total) followed by a dissolution curve.

Western blotting. Total protein from the treated BM-EPCs was extracted using a cell lysis buffer for Western and IP with proteinase inhibitors (Beyotime Institute of Biotechnology, Inc.). The concentration of total protein was determined using a BCA kit (cat. no. 23227; Thermo Fisher Scientific, Inc.). Then, $40 \mu$ g protein was separated using $10 \%$ sodium dodecyl sulfate-polyacrylamide gel electrophoresis (SDS-PAGE) and transferred to polyvinylidene fluoride membranes (EMD Millipore). The membranes were blocked with 5\% milk for $2 \mathrm{~h}$ at room temperature, then blotted with primary antibodies at $4^{\circ} \mathrm{C}$ overnight, followed by blotting with specific HRP-conjugated AffiniPure goat anti-rabbit secondary antibodies (cat. no. BA1054; Boster Biological Technology) at $37^{\circ} \mathrm{C}$ for $1 \mathrm{~h}$ after TBST (TBS with $0.05 \%$ Tween-20) washing. Finally, protein bands were visualized using enhanced chemiluminescence reagents (Pierce; Thermo Fisher Scientific, Inc.). on a Gel Imaging System (cat. no. 1708370; Edwards Life Sciences). The primary antibodies used were: Anti-GAPDH (1:10,000; RC-5G5, Kangcheng), anti-CXCR4 (1:1,000; cat. no. PA1237; Boster Biological Technology), anti-PI3K (1:1,000; cat. no. A01091-1; Boster Biological Technology) and anti-Akt (1:1,000; cat. no. A00024;Boster Biological Technology).

Statistical analysis. All data are presented as mean \pm standard deviation (SD). Statistical analysis was performed using SPSS software (version 13.0; SPSS, Inc.). One-way analysis of variance (ANOVA) was used for data with $n<50$. The least significant difference method was employed to compare samples when the variance was homogeneous. The Dunnett T3 test was used to compare samples when variance was not homogeneous. $\mathrm{P}<0.05$ was considered to indicate a statistically significant difference.

\section{Results}

Phenotype identification of BM-EPCs. The phenotype of BM-EPCs was identified through the application of immunofluorescent staining with Dil-ac-LDL and FITC-UEA-1. As depicted in Fig. 1A, the cell nuclei of BM-EPCs were blue after staining with DAPI. Endocytic vesicles were fluorescent red around the nucleus within the cytoplasm because of Dil-ac-LDL. The cell membrane was green after staining with FITC-UEA-1. The aforementioned results indicate that the expression of the EPC-specific surface marker CD14 was negative, while that of CD34 and KDR was positive (Fig. 1B).

CXCR4 is overexpressed in BM-EPCs. In order to investigate the mechanism as well as the functions associated with CXCR4 in BM-EPCs, adenoviruses were constructed (rAAV6-GFP and rAAV6-CXCR4), and the empty vector or adenovirus-CXCR4 were transfected into BM-EPCs. CXCR4 
A

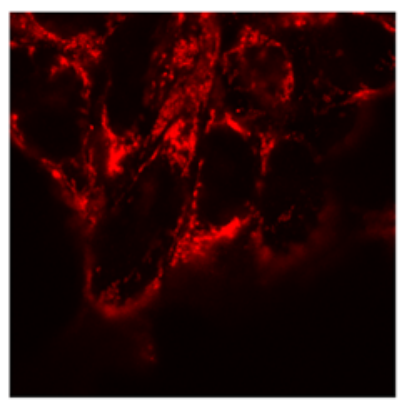

FITC-UEA-1

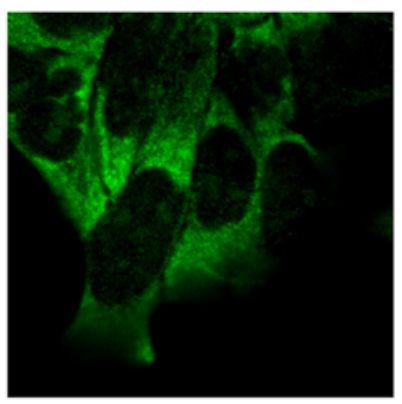

DAPI

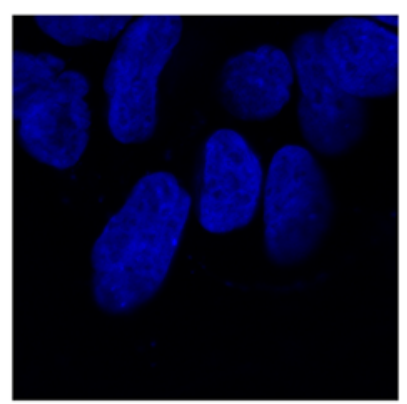

CD14

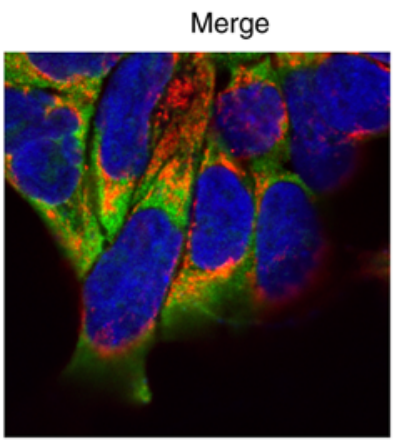

CD34

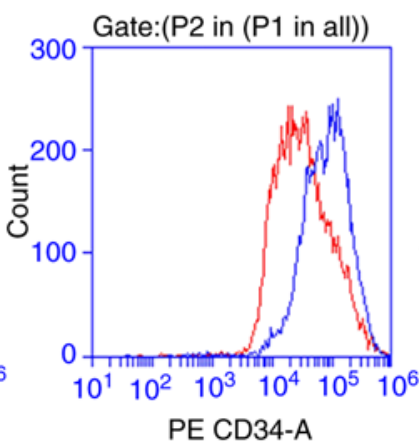

KDR

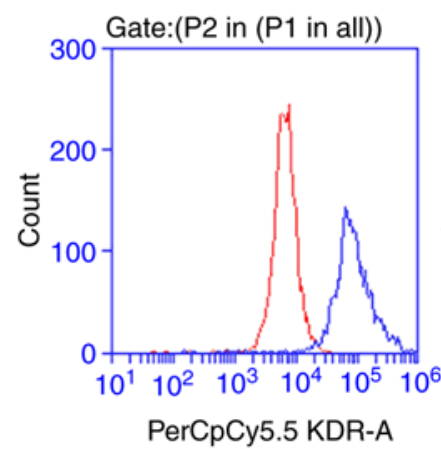

PE CD34-A

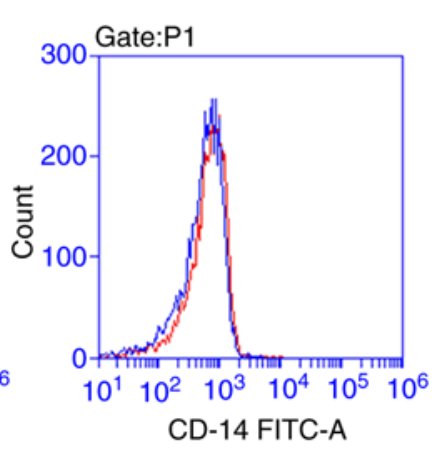

CXCR4

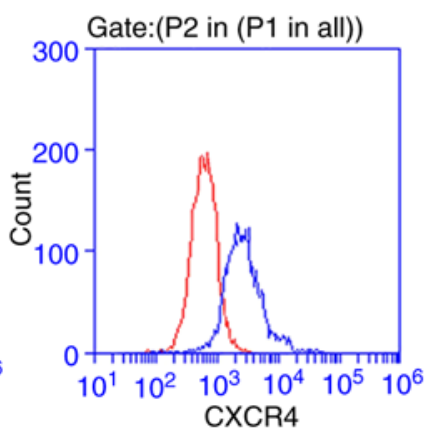

Figure 1. Phenotype identification of BM-EPCs observed following IF staining. (A) Dil-ac-LDL- and FITC-UEA-1-positive levels measured using an IF assay on BM-EPCs under a fluorescence microscope (magnification, x120). (B) CD14, CD34 and KDR detected by flow cytometry, and the levels of CXCR4 expression in BM-EPCs that were transfected with empty vector or adenovirus-CXCR4 detected by flow cytometry. BM-EPCs, bone marrow-derived endothelial progenitor cells; DiI-ac-LDL, dil-labelled acetylated low-density lipoprotein; FITC-UEA-1, fluorescein isothiocyanate labeled ulex europaeus lectin-1; DAPI, 4'6-diamidino-2-phenylindole. Immunofluorescence; CXCR4, chemokine receptor-4.

was stably overexpressed in BM-EPCs, as measured through flow cytometry assays (Fig. 1B). The mRNA and protein expression of CXCR4 was determined by RT-qPCR and western blot assays, respectively. The RT-qPCR results showed that CXCR4 mRNA was highly expressed in the CXCR4 group compared with the vector group $(\mathrm{P}<0.01$; Fig. $2 \mathrm{~A})$. Western blotting results revealed that $\mathrm{CXCR} 4$ protein was also highly expressed in the CXCR4 group compared with the vector group (Fig. 2B). The aforementioned results confirmed efficient transfection, and subsequent high expression of adenovirus-CXCR4 in BM-EPCs.

CXCR4 promotes proliferation and migration, while inhibiting apoptosis of hypoxia-induced BM-EPCs. In order to demonstrate the functional mechanism of CXCR4 in BM-EPCs under hypoxic conditions, BM-EPCs were treated with Hypoxia + CXCR4, Normoxia + CXCR4, Hypoxia + Sham (Empty vector), or Normoxia + Sham. Initially, western blotting revealed that the expression level of CXCR4, PI3K and Akt were all significantly elevated in the Normoxia + CXCR4 group compared with the Normoxia + Sham group $(\mathrm{P}<0.01)$. The expression level of CXCR4, PI3K and Akt were also significantly increased in the Hypoxia + CXCR4 group vs. the Hypoxia + Sham group $(\mathrm{P}<0.01$; Fig. 3A). Thus, it was concluded that CXCR4 upregulated the expression level of PI3K and Akt in BM-EPCs under hypoxic conditions. Moreover, the present results indicated that cell proliferation ability was significantly strengthened in the Normoxia + CXCR4 group compared with the Normoxia + Sham group $(\mathrm{P}<0.01)$, while a dramatic increase was observed

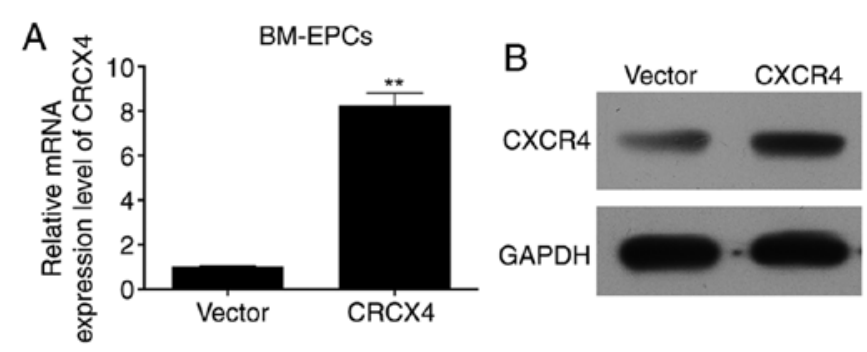

Figure 2. CXCR4 is overexpressed in BM-EPCs. BM-EPCs were transfected with empty vector or adenovirus-CXCR4. (A) The mRNA expression level of CXCR4 was measured by reverse transcription-quantitative PCR assay in BM-EPCs ( ${ }^{* *} \mathrm{P}<0.01$ vs. vector group). (B) The protein expression level of CXCR4 was examined by western blotting assay in BM-EPCs. BM-EPCs, bone marrow-derived endothelial progenitor cells; CXCR4, chemokine receptor-4.

in the Hypoxia + CXCR4 group compared with the Hypoxia + Sham group $(\mathrm{P}<0.05)$. In addition, cell proliferation ability was significantly inhibited in the Hypoxia + Sham group compared with the Normoxia + Sham group, and was also markedly suppressed in the Hypoxia + CXCR4 group compared with the Normoxia + CXCR4 group ( $\mathrm{P}<0.01$; Fig. $3 \mathrm{~B})$.

The aforementioned results suggest that hypoxia inhibits cell proliferation of BM-EPCs, while CXCR4 promotes the proliferation ability of BM-EPCs with or without hypoxia treatment. Transwell assays further indicated that hypoxia resulted in the inhibition of BM-EPC cell migration, in addition to demonstrating that CXCR4 significantly enhanced the migration ability of BM-EPCs with or without hypoxia 
treatment $(\mathrm{P}<0.01$; Fig. $3 \mathrm{C}$ and $\mathrm{E})$. Furthermore, hypoxia markedly accelerated cell apoptosis of BM-EPCs, and it was found that CXCR4 markedly suppressed the apoptosis ability of BM-EPCs with or without hypoxia treatment $(\mathrm{P}<0.01$; Fig. 3D and F).

PI3K inhibitor and AMD3100 suppresses proliferation and migration, and accelerates apoptosis of CXCR4-mediated $B M-E P C s$ under the hypoxic condition. Based on the observation that CXCR4 could upregulate the expression levels of PI3K and Akt in BM-EPCs, it was subsequently hypothesized that CXCR4 is implicated in the biological process of BM-EPCs by regulating the PI3K/Akt signaling pathway. In order to test the hypothesis, BM-EPCs were treated with Hypoxia + Sham, Hypoxia + CXCR4, Hypoxia + CXCR4 + PI3K inhibitor (LY294002), or Hypoxia + CXCR4 + CXCR4 inhibitor (AMD3100). The CCK-8 assay indicated that cell proliferation ability was significantly increased in the Hypoxia + CXCR4 group compared with the Hypoxia + Sham group $(\mathrm{P}<0.01)$. A significant decrease in cell proliferation ability was identified in the Hypoxia + CXCR4 + LY294002 group compared with the Hypoxia + CXCR4 group $(\mathrm{P}<0.05)$, and similar results were observed in the Hypoxia + CXCR4 + AMD3100 group compared with the Hypoxia + CXCR4 group ( $\mathrm{P}<0.01$; Fig. 4A).

The aforementioned findings suggest that CXCR4 functioning on the EPC surface promoted BM-EPC proliferation under hypoxic conditions, and that the PI3K inhibitor acts to attenuate the stimulation mediated by CXCR4 in BM-EPCs under hypoxic conditions. Transwell assays further verified that CXCR4 significantly accelerated the migration of BM-EPCs under hypoxic conditions, while illustrating that PI3K inhibitor and CXCR4 inhibitor weakened the acceleration mediated by CXCR4 in BM-EPCs under hypoxic conditions ( $\mathrm{P}<0.01$; Fig. 4B and D). More importantly, CXCR4 significantly repressed the apoptosis of BM-EPCs under hypoxic conditions, but PI3K inhibitor or CXCR4 inhibitor blocked the inhibition mediated by CXCR4 in BM-EPCs under hypoxic conditions $(\mathrm{P}<0.01$; Fig. $4 \mathrm{C}$ and $\mathrm{E})$. These findings have significant implications on the understanding of how CXCR4 promotes the proliferation and migration of BM-EPCs, and suppresses their apoptosis through the PI3K signaling pathway.

\section{Discussion}

The pathogenesis of the majority of CVDs involves vascular endothelial damage (13), and hypoxia represents a chief factor implicated in vascular endothelial damage (14). Therefore, strategies to prevent vascular endothelial cell injury could potentially decrease the occurrence and severity of CVDs. The present study focused on the role of CXCR4 in protecting BM-derived EPCs in hypoxia, and found that the overexpression of CXCR4 promoted the proliferation and migration abilities of hypoxia-induced BM-EPCs and simultaneously inhibited the apoptosis ability of hypoxia-induced BM-EPCs through the PI3K/Akt signaling pathway.

Firstly, mononuclear cells were successfully isolated from the bone marrow of rats under a hypoxic environment and EPCs were characterized by immunofluorescence assay with
DIL-ac-LDL and FITC-UEA-1 labeling, resulting in cells that were CD14-negative, CD34- and KDR-positive, suggesting that EPCs have similar characteristics to endothelial cells. EPCs are defined as precursors of endothelial cells with positive expression of CD34, CD133 and VEGFR2 (15). EPCs are also BM mononuclear cells with partial endothelial function and differentiation ability, which can bind to ulex europaeus agglutinin-1 (UEA-1) and acetylated low-density lipoprotein (AcLDL) (16). EPCs derived from BM possess the ability to proliferate, migrate and differentiate into mature endothelial cells, as well as participate in angiogenesis, bind to blood vessels, and stimulate the proliferation of neighboring endothelial cells (17). Studies have highlighted the key role of EPCs in CVDs (18). Therefore, CXCR4 may be a potential therapeutic target for vascular damage and regeneration.

Secondly, CXCR4 was found to protect BM-derived EPCs under hypoxia. CXCR4, as a receptor for SDF-1, represents a crucial factor in the regulatory process of EPC homing (19). The blockade of CXCR4 signaling was previously shown to alleviate the decreased homing of EPCs to injured arteries (20), indicating that CXCR4 signaling may be a potential molecular target for gene therapy associated with the dysfunction of EPCs. The SDF-1/CXCR4 signaling pathway plays a key role in cell proliferation, tumor metastasis, angiogenesis, and the reendothelialization capacity of EPCs $(21,22)$. The SDF-1 $\alpha /$ CXCR4 axis depletes EPC apoptosis under serum deprivation conditions through the PI3K/Akt signaling pathway (23), but the functions and mechanisms of CXCR4 in ischemic vascular diseases remain unknown. Adenoviruses were constructed (rAAV6-GFP and rAAV6-CXCR4), and successfully transfected into BM-EPCs. It was found that overexpression of CXCR4 promoted the proliferation and migration abilities of hypoxia-induced BM-EPCs. Simultaneously, the overexpression of CXCR4 inhibited the apoptosis ability of hypoxia-induced BM-EPCs. Therefore, CXCR4 may serve as a potential therapeutic target for the regeneration of impaired blood vessels.

Thirdly, CXCR4 upregulates the expression level of the PI3K and Akt signaling pathway in BM EPCs under hypoxic conditions; PI3K inhibitor (ly294002) inhibits proliferation and migration under hypoxia, and promotes the apoptosis of BM-EPC mediated by CXCR4. The PI3K signaling pathway is activated by various cellular stimuli or toxic insults, and induces the phosphorylation of key downstream effectors such as Akt. The activation of PI3K leads to an increase in phosphatidylinositol-(3,4,5)-triphosphate (PIP3) (24), which can bind to the pleckstrin homology on PDK1. Then, PDK1 can phosphorylate Akt at the activation loop (25). Akt phosphorylates several antiapoptotic proteins, cell-cycle-related proteins and transcription factors $(24,25)$. Akt can regulate intracellular signals, affect cell responses to extrinsic stimuli, and participate in cell proliferation and survival (26). Extensive research has shown that PI3K/Akt are involved in an array of growth factor signal transduction pathways, and exert multiple biological functions (27). The present study found that CXCR4 upregulates PI3K and Akt expression in BM-EPCs under hypoxic conditions. In addition, PI3K inhibitor (LY294002) inhibits proliferation and migration, while promoting the apoptosis of CXCR4-mediated BM-EPCs under hypoxic conditions.

CXCR4 has been found to be expressed in $>23$ different types of cancer (28). SDF-1/CXCR4 is involved in breast cancer, 
A

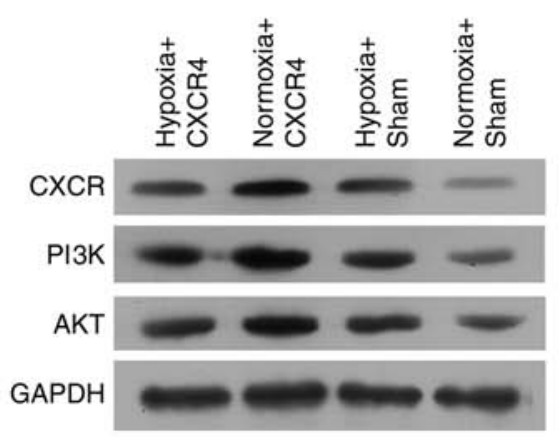

C

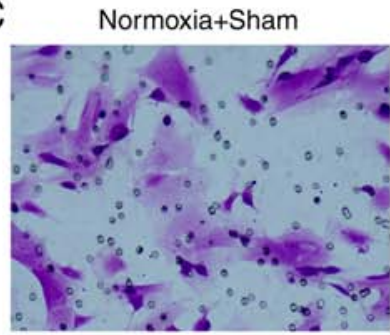

D Normoxia+Sham

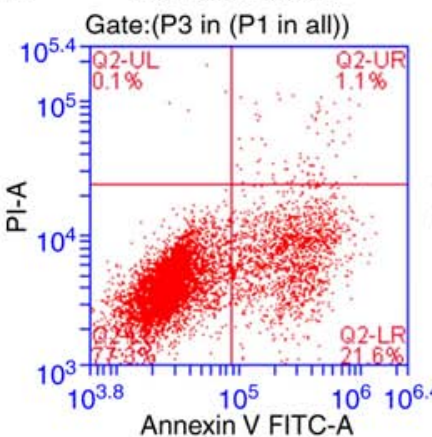

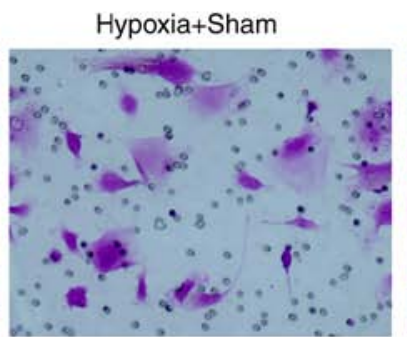

Hypoxia+Sham

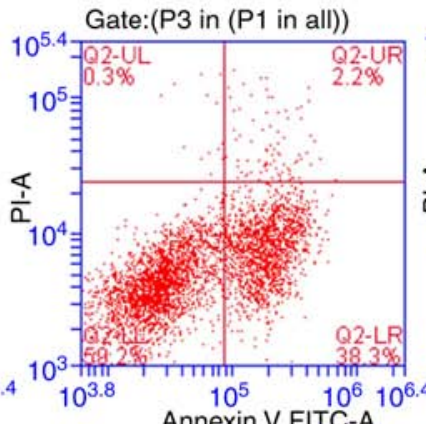

Annexin V FITC-A
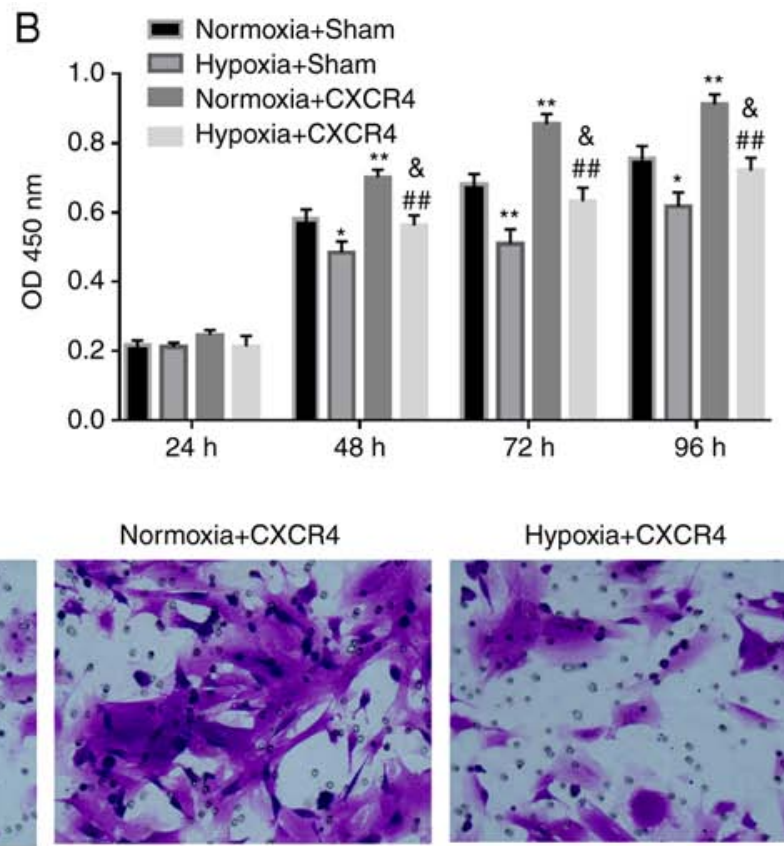

Normoxia+CXCR4

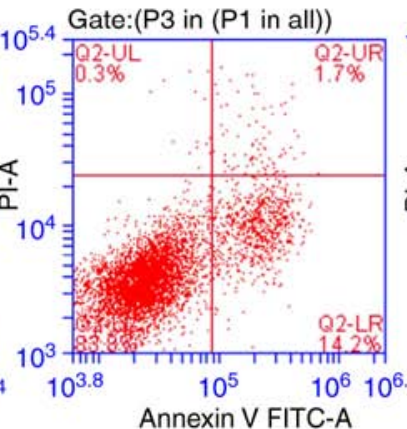

Hypoxia+CXCR4

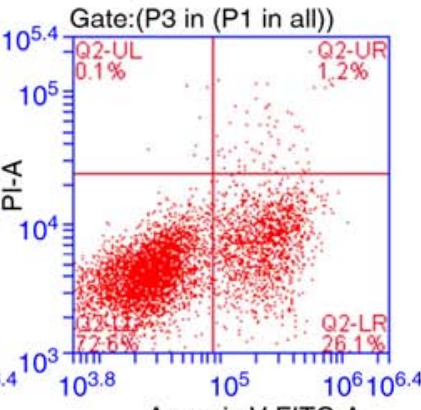

Annexin V FITC-A
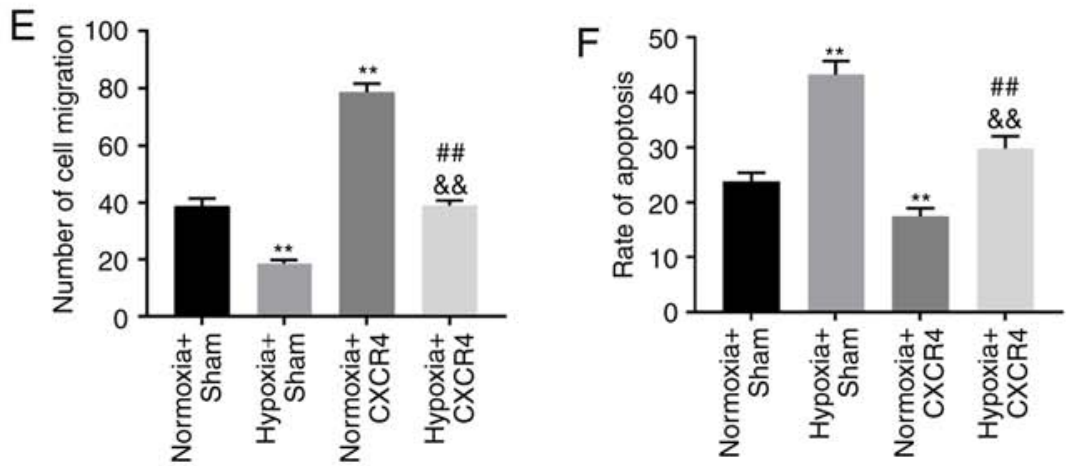

Figure 3. CXCR4 promotes proliferation and migration, and inhibits apoptosis of hypoxia-induced BM-EPCs. BM-EPCs were divided into four groups: Hypoxia + CXCR4, Normoxia + CXCR4, Hypoxia + Sham, and Normoxia + Sham groups. (A) The protein expression levels of CXCR4, PI3K and AKT were measured by western blotting. GAPDH was regarded as the loading control. (B) Cell proliferation ability detected by Cell Counting Kit-8 assay. (C) Migration capacity was determined by Transwell assay (magnification, x200). (D) Apoptosis capacity was detected by flow cytometry. (E) Quantification of CXCR4-promoted migration of hypoxia-induced BM-EPCs. (F) Quantification of CXCR4-inhibited apoptosis in hypoxia-induced BM-EPCs. Experiments were repeated 3 times; data are expressed as mean $\pm \mathrm{SD}$. ${ }^{*} \mathrm{P}<0.05$ and ${ }^{* *} \mathrm{P}<0.01$ vs. Normoxia + Sham; ${ }^{\circledR} \mathrm{P}<0.05$ and ${ }^{\& \&} \mathrm{P}<0.01$ vs. Hypoxia + Sham; ${ }^{\# \#} \mathrm{P}<0.01$ vs. Normoxia $+\mathrm{CXCR} 4$. BM-EPCs, bone marrow-derived endothelial progenitor cells; PI3K, phosphatidylinositol 3-kinase; GAPDH, glyceraldehyde-3-phosphate dehydrogenase; CXCR4, chemokine receptor-4.

glioma and neuroblastoma, which promotes endothelial progenitor cells homing to form neovascularization (29-31). In the present study, CXCR4-overexpressing EPCs showed enhanced cellular proliferation and decreased apoptosis under hypoxia, as well as normoxia. The intrinsic increase in PI3K/Akt is one of the major causes of transformation. Therefore, the potential risk of tumors and hemangiomas caused by CXCR4-overexpressing EPCs should be carefully considered.

A limitation of the present study was that there were no set gradient values for SDF-1, LY294002 and AMD3100; thus, the most effective relative concentration for the interaction between SDF-1 and CXCR4, and the relative concentration 


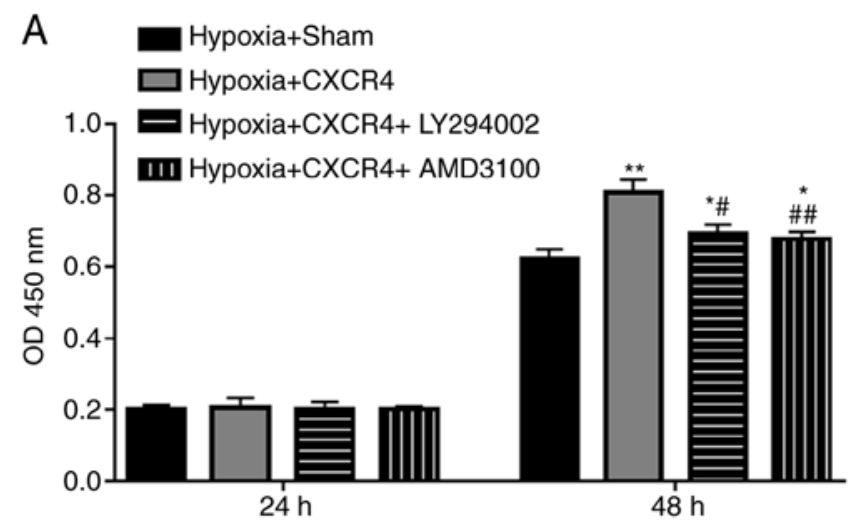

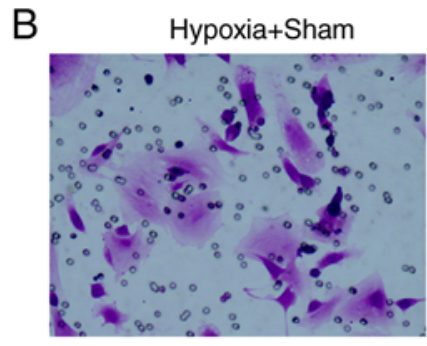
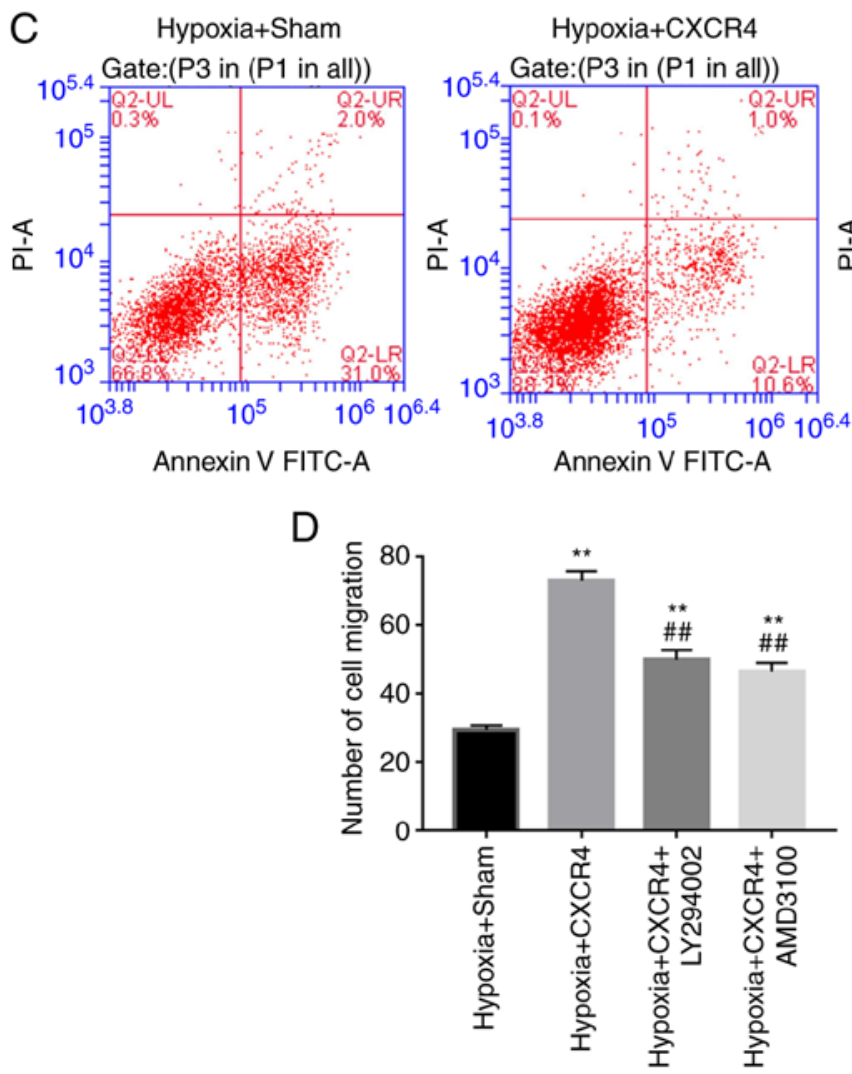

Hypoxia+CXCR4

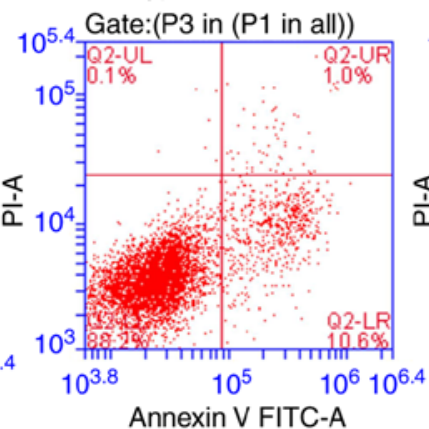

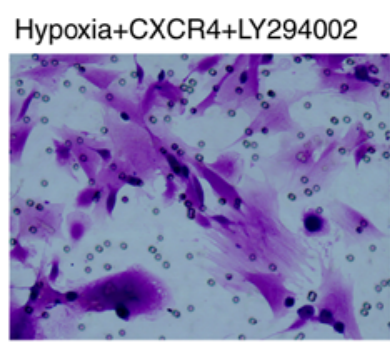

Hypoxia+CXCR4+ LY294002

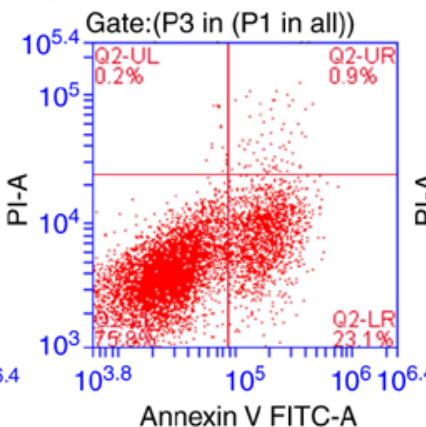

$\mathrm{E}$

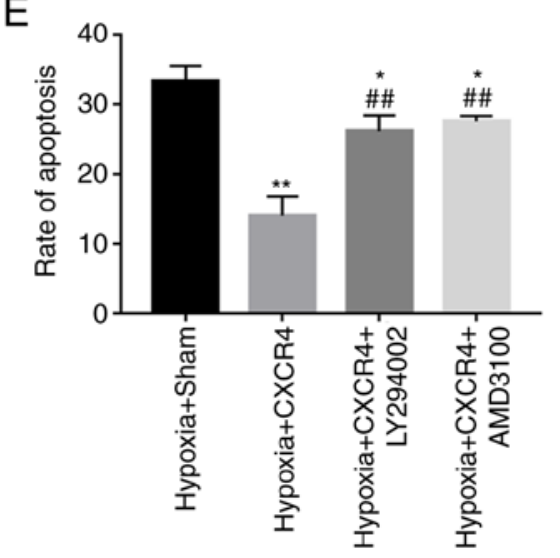

Figure 4. PI3K inhibitor or CXCR4 inhibitor suppresses proliferation and migration, while accelerating apoptosis of CXCR4-mediated BM-EPCs under hypoxic conditions. BM-EPCs were divided into four groups: Hypoxia + Sham, Hypoxia + CXCR4, Hypoxia + CXCR4 + LY294002, and Hypoxia + CXCR4 + AMD3100 groups. (A) Cell Counting Kit-8 assay was used to assess the proliferation abilities of BM-EPCs. (B) Transwell assay to evaluate cell migration ability (magnification, x200). (C) Flow cytometry to analyze cell apoptosis capacity. (D) Quantification of LY294002- and AMD3100-suppressed migration of CXCR4-mediated BM-EPCs under hypoxic conditions. (E) Quantification of LY294002 and AMD3100 increased apoptosis of CXCR4-mediated BM-EPCs under hypoxic conditions. Experiments were repeated 3 times; data are expressed as mean $\pm \mathrm{SD}$. ${ }^{*} \mathrm{P}<0.05$ and ${ }^{* *} \mathrm{P}<0.01$ vs. Hypoxia + Sham; ${ }^{\#} \mathrm{P}<0.05$ and ${ }^{\# \# /} \mathrm{P}<0.01$ vs. Hypoxia + CXCR4. BM-EPCs, bone marrow-derived endothelial progenitor cells; PI3K, phosphatidylinositol 3-kinase; CXCR4, chemokine receptor-4.

of CXCR4 inhibition by LY294002 and AMD3100 were unknown.

In conclusion, the present study suggests that CXCR4 accelerates proliferation and migration, and suppresses apoptosis of hypoxia-induced BM-EPCs through the PI3K signaling pathway in rats. These results open new avenues of research to better understand the role of BM-EPCs in vascular repair. 


\section{Acknowledgements}

Not applicable.

\section{Funding}

This study was supported by the Outstanding Youth Project of the Education Bureau of Hunan Province (grant no. 18B036), the Natural Science Foundation of Hunan Province (grant no. 2020JJ4406), the Type B vertical project of the Health and Family Planning Commission (grant no. 20190489), the Hunan Natural Science Foundation joint project of the Science and Technology Commission (grant no. 2020JJ8082), and the Scientific Research Project of the Hunan Provincial Health Commission (grant no. B2019066).

\section{Availability of data and materials}

The datasets used and/or analyzed during the current study are available from the corresponding author on reasonable request.

\section{Authors' contributions}

ZYL, ZFZ, BLZ and QXY conceived and designed the study; YC, JJR, JL, YT, CLW, XP, QCZ, LZ, LBX, FL and ZHY acquired the experimental data; HWP, JH, JZ, JW, YZ, JQP and ZFZ analyzed and interpreted the data; and ZYL, QXY and BLZ drafted the article or revised it critically for intellectual content. ZFZ and BLZ agree to be accountable for all aspects of the work, ensuring that questions related to the accuracy or integrity of any part of the work are appropriately investigated and resolved. ZYL, ZFZ, BLZ and QXY were responsible for confirming the authenticity of all the raw data, All authors have read and approved the final version of the manuscript.

\section{Ethics approval and consent to participate}

This study was conducted with the approval of the Ethics Committee of the Hunan Provincial People's Hospital (Changsha, China) [approval no. (2019)-041]. The animals used in this study were cared for in accordance with the Guide for the Care and Use of Laboratory Animals published by the National Institutes of Health. All efforts were made to minimize the suffering of the animals included in the study.

\section{Patient consent for publication}

Not applicable.

\section{Competing interests}

The authors declare that they have no competing interests.

\section{References}

1. GBD 2016 Causes of Death Collaborators: Global, regional, and national age-sex specific mortality for 264 causes of death, 1980-2016: A systematic analysis for the global burden of disease study 2016. Lancet 390: 1151-1210, 2017.

2. Townsend N, Wilson L, Bhatnagar P, Wickramasinghe K, Rayner M and Nichols M: Cardiovascular disease in Europe: Epidemiological update 2016. Eur Heart J 37: 3232-3245, 2016.
3. Ramakrishnan S, Anand V and Roy S: Vascular endothelial growth factor signaling in hypoxia and inflammation. J Neuroimmune Pharmacol 9: 142-60, 2014

4. Jaipersad AS, Lip GY, Silverman S and Shantsila E: The role of monocytes in angiogenesis and atherosclerosis. J Am Coll Cardiol 63: 1-11, 2014.

5. Sharifpanah F, Behr S, Wartenberg M and Sauer H: Mechanical strain stimulates vasculogenesis and expression of angiogenesis guidance molecules of embryonic stem cells through elevation of intracellular calcium, reactive oxygen species and nitric oxide generation. Biochim Biophys Acta 1863: 3096-3105, 2016

6. Rafii S, Butler JM and Ding BS: Angiocrine functions of organ-specific endothelial cells. Nature 529: 316-325, 2016.

7. Döring Y, Pawig L, Weber C and Noels H: The CXCL12/CXCR4 chemokine ligand/receptor axis in cardiovascular disease. Front Physiol 5: 212, 2014.

8. Schober A and Zernecke A: Chemokines in vascular remodeling. Thromb Haemost 97: 730-737, 2007.

9. Jacobson $O$ and Weiss ID: CXCR4 chemokine receptor overview: Biology, pathology and applications in imaging and therapy. Theranostics 3: 1-2, 2013.

10. Yamaguchi J, Kusano KF, Masuo O, Kawamoto A, Silver M, Murasawa S, Bosch-Marce M, Masuda H, Losordo DW, Isner JM and Asahara T: Stromal cell-derived factor-1 effects on ex vivo expanded endothelial progenitor cell recruitment for ischemic neovascularization. Circulation 107: 1322-1328, 2003.

11. Segal MS, Shah R, Afzal A, Perrault CM, Chang K, Schuler A, Beem E, Shaw LC, Li Calzi S, Harrison JK, et al: Nitric oxide cytoskeletal-induced alterations reverse the endothelial progenitor cell migratory defect associated with diabetes. Diabetes 55: 102-109, 2006.

12. Navidshad B, Liang JB and Jahromi MF: Correlation coefficients between different methods of expressing bacterial quantification using real time PCR. Int J Mol Sci 13: 2119-2132, 2012.

13. Matsuzawa Y, Guddeti RR, Kwon TG, Lerman LO and Lerman A: Treating coronary disease and the impact of endothelial dysfunction. Prog Cardiovasc Dis 57: 431-442, 2015.

14. Dai Z, Li M, Wharton J, Zhu MM and Zhao YY: Prolyl-4 hydroxylase 2 (PHD2) deficiency in endothelial cells and hematopoietic cells induces obliterative vascular remodeling and severe pulmonary arterial hypertension in mice and humans through hypoxia-inducible factor-2 $\alpha$. Circulation 133: 2447-2458, 2016.

15. Cesari F, Sofi F, Molino Lova R, Vannetti F, Pasquini G, Cecchi F, Marcucci R, Gori AM and Macchi C; Mugello Study Working Group: Aging process, adherence to mediterranean diet and nutritional status in a large cohort of nonagenarians: Effects on endothelial progenitor cells. Nutr Metab Cardiovasc Dis 28: 84-90, 2018.

16. Dai X, Yan X, Zeng J, Chen J, Wang Y, Chen J, Li Y, Barati MT, Wintergerst KA, Pan K, et al: Elevating CXCR7 improves angiogenic function of EPCs via Akt/GSK-3ß/Fyn-mediated Nrf2 activation in diabetic limb ischemia. Circ Res 120: e7-e23, 2017.

17. Bianconi V, Sahebkar A, Kovanen P, Bagaglia F, Ricciuti B, Calabrò $\mathrm{P}$, Patti G and Pirro M: Endothelial and cardiac progenitor cells for cardiovascular repair: A controversial paradigm in cell therapy. Pharmacol Ther 181: 156-168, 2018.

18. Werner N, Kosiol S, Schiegl T, Ahlers P, Walenta K, Link A, Böhm M and Nickenig G: Circulating endothelial progenitor cells and cardiovascular outcomes. N Engl J Med 353: 999-1007, 2005.

19. Napoli C, Hayashi T, Cacciatore F, Casamassimi A, Casini C, Al-Omran M and Ignarro LJ: Endothelial progenitor cells as therapeutic agents in the microcirculation: An update. Atherosclerosis 215: 9-22, 2011.

20. Wara AK, Manica A, Marchini JF, Sun X, Icli B, Tesmenitsky Y, Croce $\mathrm{K}$ and Feinberg MW: Bone marrow-derived Kruppel-like factor 10 controls reendothelialization in response to arterial injury. Arterioscler Thromb Vasc Biol 33: 1552-1560, 2013.

21. Ruiz A, Ruiz L, Colón-Caraballo M, Torres-Collazo BJ, Monteiro JB, Bayona M, Fazleabas AT and Flores I: Pharmacological blockage of the CXCR4-CXCL12 axis in endometriosis leads to contrasting effects in proliferation, migration, and invasion. Biol Reprod 98: 4-14, 2018.

22. Bianchi ME and Mezzapelle R: The chemokine receptor CXCR4 in cell proliferation and tissue regeneration. Front Immunol 11: 2109, 2020

23. Zheng H, Dai T, Zhou B, Zhu J, Huang H, Wang M and Fu G: SDF-1alpha/CXCR4 decreases endothelial progenitor cells apoptosis under serum deprivation by PI3K/Akt/eNOS pathway. Atherosclerosis 201: 36-42, 2008. 
24. Manning BD and Toker A: AKT/PKB signaling: Navigating the network. Cell 169: 381-405, 2017.

25. Walton KL, Holt L and Sartor RB: Lipopolysaccharide activates innate immune responses in murine intestinal myofibroblasts through multiple signaling pathways. Am J Physiol Gastrointest Liver Physiol 296: G601-G611, 2009.

26. Wang YQ, Zhang JR, Li SD, He YY, Yang YX, Liu XL and Wan XP: Aberrant methylation of breast and ovarian cancer susceptibility gene 1 in chemosensitive human ovarian cancer cells does not involve the phosphatidylinositol 3'-kinase-Akt pathway. Cancer Sci 101: 1618-1623, 2010.

27. Polivka J Jr and Janku F: Molecular targets for cancer therapy in the PI3K/AKT/mTOR pathway. Pharmacol Ther 142: 164-175, 2014.

28. Zhou W, Guo S, Liu M, Burow ME and Wang G: Targeting CXCL12/CXCR4 axis in tumor immunotherapy. Curr Med Chem 26: 3026-3041, 2019.

29. Orimo A, Gupta PB, Sgroi DC, Arenzana-Seisdedos F, Delaunay $T$, Naeem R, Carey VJ, Richardson AL and Weinberg RA: Stromal fibroblasts present in invasive human breast carcinomas promote tumor growth and angiogenesis through elevated SDF-1/CXCL12 secretion. Cell 121: 335-348, 2005 .
30. Cheng L, Huang Z, Zhou W, Wu Q, Donnola S, Liu JK, Fang X, Sloan AE, Mao Y, Lathia JD, et al: Glioblastoma stem cells generate vascular pericytes to support vessel function and tumor growth. Cell 153: 139-152, 2013

31. Du R, Lu KV, Petritsch C, Liu P, Ganss R, Passegué E, Song H, Vandenberg S, Johnson RS, Werb Z and Bergers G: HIFlalpha induces the recruitment of bone marrow-derived vascular modulatory cells to regulate tumor angiogenesis and invasion. Cancer Cell 13: 206-220, 2008.

(i) (8) This work is licensed under a Creative Commons Attribution 4.0 International (CC BY-NC 4.0) License 